\title{
Efficacité de l'utilisation de la stratégie d'auto-questionnement sur le développement des compétences de la compréhension écrite chez les étudiants du cycle secondaire des écoles de langue
}

\author{
Dalia Mahmoud Mohammed Hamed
}

\section{Introduction}

La langue joue un rôle vital à renforcer les relations dans la vie des individus et des sociétés. L'homme utilise la langue pour communiquer et exprimer ses besoins, ses ambitions, ses rêves et ses sentiments (joie, malheur, surprise, admiration). ...etc.

Les langues étrangères jouent un rôle primordial à ouvrir les horizons des apprenants sur le monde entier. C'est pour cela qu'on accorde à l'enseignement des langues étrangères une grande importance afin de rendre les étudiants capables de communiquer et de s'adapter aux nouveaux changements dans tous les domaines.

La langue est un moyen de communication entre les peuples. Selon Soleiman (1996: 2) " $L a$ langue est donc un moyen de communication qui comporte 4 compétences langagière: Entendre et Comprendre, Parler, Lire et Ecrire.

L'apprentissage/l'enseignement d'une langue étrangère doit donc prendre en considération ces 4 compétences comme unité intégrante sans négliger l'une ou l'autre".

Pour apprendre une langue l'apprenant a besoin de développer les quatre coméptences fondamentales suivantes: $\mathrm{La}$ compréhension orale, l'expression orale, la compréhension écrite et l'expression écrite. Parmi ces compétences, on s'intéresse pendant cette recherche à la compréhension écrite qui est le chemin de l'homme pour savoir ce qui l'entoure. Cette compétence est aussi la source de la connaissance.

Selon Cuq (2003: 49), "la compréhension est l'aptitude résultant de la mise en ouvre de processus cognitifs, qui permet à l'apprenant d'accéder au sens d'un texte qu'il lit".

La compréhension des textes est le but essentiel de la lecture. La compréhension d'un document écrit est un processus interactif qui met en relation un lecteur et un texte dans un contexte. Dans ce 
processus, le lecteur crée le sens en se servant à la fois du texte, de ses propres connaissances et de son intention de lecture. Giasson (2000: $5)$.

Selon Afifi (2018: 4), la compréhension écrite exige les quatre étapes suivantes:

- La planification (cadrer): choisir un cadre cognitif, mettre en oeuvre un schéma et créer des attentes.

- L'exécution: identifier des indices et en tirer une déduction.

- L'évaluation (vérifier des hypothèses): apparier les indices et le schéma.

- La remédiation: réviser les hypothèses s'il y a lieu

La compréhension écrite est une compétence linguistique très importante qui aide à développer chez l'apprenant la capacité à réfléchir et à comprendre. On lit donc pour développer sa personnalité, pour élargir ses expériences et ses connaissances et pour savoir ce qui se passé autour de soi.

Dans nos jours et avec le progrès scientifique, il est nécessaire d'utiliser de nouvelles stratégies visant à développer les compétences de la compréhension écrite. C'est pourquoi la chercheuse a adopté la stratégie d'autoquestionnement qui aide à développer ces compétences chez les étudiants des écoles de langue.

\section{Cette} stratégie

d'enseignement permet aux apprenants d'apprendre en se questionnant, en se parlant intérieurement et en trouvant des réponses à leurs questions de manière individuelle.

L'autoquestionnement est différent des approches de questionnements traditionnels car il permet à l'étudiant de jouer un rôle actif dans la démarche de questionnement. Ainsi, les adeptes de l'autoquestionnement proposent que les formateurs enseignent aux étudiants à se poser eux même des questions. Viola (2000: 2)

Cette stratégie doit être enseignée aux étudiants en tout début d'apprentissage comme suit:

- La première étape consiste à identifier les questions que l'étudiant doit arriver à se poser afin de les aider à bien s'autoquestionner. Ces questions peuvent être de types cognitifs, métacognitifs et affectifs.

- La deuxième étape est d'aider les étudiants à identifier les bons moments pour s'autoquestionner. C'est-à-dire avant, pendant et après la lecture. On peut donc proposer aux étudiants un schéma de temps pour qu'ils structurent 
leurs autoquestionnements. Ce schéma peut être affichée dans la classe sous forme d'affiche ou par projection.

- Dans la troisième étape, on doit enseigner aux étudiants l'utilité et la rentabilité de la stratégie d'autoquestionnement afin d'augmenter leur intérêt à utiliser cette stratégie.

- La dernière étape consiste à apporter l'aide nécessaire aux étudiants de façon individuelle pour qu'ils puissent s'autoquestionner efficacement. C'est un cheminement qui vise à donner à l' étudiant le pouvoir sur ses apprentissages car celuici sera en mesure de questionner les textes de façon autonome. (Viola, 2000: 6).

Le rôle de l'enseignant est de former des étudiants qui sauront se questionner de façon autonome.

2. Sensibilisation à la problématique de la recherche

Malgré l'importance de la compréhension écrite comme moyen

d'apprentissage/d'enseignement de chaque langue ainsi d'adaptation à la société, il y a beaucoup de difficultés de la compréhension écrite. C'est pourquoi beaucoup d'études sont été effectuées dans le domaine de développement des compétences de la compréhension écrite comme: Elsherbini (2007),
Makhlouf (2001), Baier (2005), Fouad (2014).

Tous les résultats de ces études ont constaté qu':

- Il existe une faiblesse
remarquable chez les étudiants
en ce qui concerne les
compétences de
compréhension écrite.
- Il est nécessaire d'utiliser de
nouvelles stratégies et
méthodes pour développer les
compétences de
compréhension écrite.

\section{Problématique de la recherche}

La recherche actuelle essaie de répondre à la question principale suivante: Quelle est l'efficacité de la stratégie d'autoquestionnement sur le développement des compétences de la compréhension écrite chez les étudiants des écoles de langue du cycle secondaire?

Afin de répondre à cette question, il s'avère nécessaire de répondre aux sous-questions suivantes:

- Quelles sont les compétences de la compréhension écrite nécessaires aux étudiants des écoles de langue du cycle secondaire?

- A quel point les étudiants des écoles de langue du cycle secondaire possèdent - ils ces compétences? 
- Quelle est l'efficacité de l'emploi de la stratégie d'autoquestionnement sur le développement des compétences de la compréhension écrite?

\section{Objectifs de la recherche}

La recherche actuelle vise à:

- Déterminer les compétences de la compréhension écrite nécessaires aux étudiants des écoles de langue du cycle secondaire.

- Préciser l'efficacité de la stratégie d'autoquestionnement sur le développement des compétences de la compréhension écrite chez les étudiants du cycle secondaire des écoles de langue.

\section{Méthodologie de la recherche}

La chercheuse utilise, pendant la recherche actuelle, la méthode descriptive et la méthode quasi-expérimentale.

- Premièrement, la méthode descriptive pour ce qui concerne le cadre théorique de la recherche.

- Deuxièmement, la méthode quasi-expérimentale pour mesurer l'efficacité de l'emploi de la stratégie d'autoquestionnement afin de développer certaines compétences de la compréhension écrite chez les étudiants du cycle secondaire des écoles de langue.

6. Outils et matériels de la recherche

- Grille des compétences de la compréhension écrite nécessaires aux étudiants des écoles de langue du cycle secondaire.

- Test de mesure des compétences de la compréhension écrite chez les étudiants du cycle secondaire des écoles de langue.

\section{Hypothèse de la recherche}

La présente étude essaie de mettre en preuve l'hypothèse suivante:

- Il y a une différence significative statistiquement au niveau de $p \geq 0.05$ entre les moyennes des notes des étudiants $\mathrm{du}$ groupe expérimental au pré /post-tests en faveur du post-test.

\section{Délimites de la recherche}

Cette recherche se limite à:

- Un échantillon d'étudiants de la $1{ }^{\text {ère }}$ année des écoles officielles françaises des langues du cycle secondaire à Mansourah.

- Certaines compétences de la compréhension écrite.

- Unité reformulée selon la stratégie d'autoquestionnement.

\section{Démarches de la recherche:}


Pour répondre aux questions de la recherche, la chercheuse poursuit les étapes suivantes:

- Passer en revue les études antérieures portant sur les stratégies d'enseignement/apprentissage du FLE, surtout la stratégie d'auto-questionnement et les compétences de la compréhension écrite.

- Élaborer une grille des compétences de la compréhension écrite nécessaires aux étudiants de la 1èreannée des écoles officielles françaises des langues du cycle secondaire à Mansourah.

- Élaborer un test de mesure des compétences de la compréhension écrite chez les étudiants de la 1ère année des écoles officielles françaises de langues du cycle secondaire.

- Choisir une unité proposée et la reformuler selon la stratégie d'auto-questionnement afin de développer les compétences de la compréhension écrite chez les étudiants de la 1ère année des écoles officielles françaises des langues du cycle secondaire.

- Soumettre les outils et le matériel de la recherche au jury pour vérifier leur validité apprenante.

- Modifier les outils d'après les points de vue du jury.
- Choisir un échantillon des étudiants de la 1ère année des écoles officielles françaises des langues $\mathrm{du}$ cycle secondaire comme échantillon représentative.

- Appliquer le pre-test à l'échantillon de la recherche.

- Enseigner l'unité proposée à l'échantillon de la recherche.

- Appliquer le post-test à l'échantillon de la recherche.

- Analyser statistiquement les résultats de la recherche.

- Interpréter les résultats de la recherche.

- Présenter les recommandations et les suggestions de la recherche.

\section{Terminologies de l'étude}

\section{La compréhension écrite}

La recherche actuelle adopte la définition de Mefleh (2005: 278), dans laquelle il indique que "la compréhension écrite est un processus d'interaction entre le lecteur et le texte qui se reflète dans la capacité de comprendre, de conclure, de justifier et de contrôler".

\section{La stratégie d'auto- questionnement}

La recherche actuelle adopte la définition de Selon Théorêt et al. (2004), cité par Viola (2000: 2), l'auto-questionnement est une des stratégies métacognitives qui aide les étudiants à s'autoquestionner 
avant, pendant et après la lecture d'un texte. Ces questions peuvent être catégorisé d'ordre cognitifs, affectifs ou métacognitifs.

\section{Cadre théorique}

Le cadre theorique de cette recherche porte sur deux axes :

- Première axe:

la compréhension écrite

- Deuxieme axe: La stratégie d'auto-questionnement

\subsection{Première axe: la} compréhension écrite

\subsubsection{Notion de la lecture}

La lecture est un moyen important pour élargir les connaissances du lecteur. Pour Laforgue (1982: 12) La lecture, c'est voir et en même temps comprendre le contenu du message écrit. Comprendre ce qu'on déchiffre, c'est de faire passer directement du signe écrit à la signification. ( cité par Fouad 2014: 16).

Selon Nilsson (2006: 4), cité par Abdelaal (2014: 19), la lecture du texte en langue étrangère, ne se limite pas à la compréhension du texte lu mais suscite également de diverses réflexions et des réactions de la part du lecteur. Nous considérerons, néanmoins, que la lecture implique des processus qui contribuent à divers degrés à une compréhension du texte lu. Elle dépendrait des connaissances antérieures du lecteur et notamment des connaissances linguistiques.

Selon Turcotte (1992: 100) La lecture est ainsi une activité, cognitive et langagière, apprise, accomplie sous le contrôle d'un individu qui poursuit un but propre, elle est orientée vers la compréhension, c'est-à-dire la construction du sens du texte par le lecteur, et elle est effectuée avec une aisance et une motivation variables qui en affectent le résultat.

En fait, la lecture est un phénomène difficile pour le découvrir. On renverra à Adam que la lecture est un acte compliqué qui comprend les processus suivants. (Abdel-Ghany, 2009: 28).

D'autre part, la lecture ne signifie pas seulement la capacité à reconnaître les lettres, les mots et la prononciation des sons, mais aussi elle consiste à comprendre le sens des mots et des phrases. Elle est conçue comme activité cognitive de prise et de traitement de l'information pendant laquelle le lecteur (identifie des signes) et construit le sens. (Sobhi, 2010: 5859).

\subsubsection{Objectifs de la lecture}

La compréhension est le but de la lecture. En ce sens, la lecture a des objectifs variés soit soutenir la motivation à faire lire des étudiants, renforcer la fluidité, 
entraîner les étudiants à gérer leur compréhension des textes lus et exploiter la critique. Les descriptions suivantes associent chaque objectif à une intervention de l'enseignant et aux effets observables sur les habiletés en lecture des étudiants comme suit:

- Soutenir la motivation à faire lire des étudiants en adoptant des pratiques pédagogiques éprouvées et en mettant systématiquement la lecture au service de l'apprentissage. Ceci permet d'observer le développement d'attitudes positives par rapport à la lecture et de maintenir l'intérêt des étudiants pour cette activité et le monde de l'écrit.

- Renforcer la fluidité en exploitant des activités pertinentes qui contribuent au développement des habiletés de fluidité. Ceci permet de consolider l'habileté à reconnaître rapidement les mots à lire le texte avec précision et expression, ce qui favorise l'accès à la compréhension des textes lus.

- Entraîner les étudiants à gérer leur compréhension des textes lus en enseignant, dans des situations authentiques, des stratégies de compréhension, en faisant l'étude de vocabulaire ou des notions de grammaire et en analysant les éléments d'écriture. Ceci permet d'élargir le champ de compréhension des textes lus de tout genre.

- Exploiter la littératie critique en valorisant l'exercice des habiletés supérieures de la pensée en analysant et en évaluant avec les étudiants l'intention de l'auteur et les messages qu'il véhicule dans ses textes. Ceci permet de développer la pensée critique des étudiants. (Abdel-Aal, 2014: 21).

Savoir lire, c'est donc, pouvoir traiter de manière autonome les informations dans sa langue en vue de les comprendre. La compréhension est le but de la lecture, le traitement du contenu est le moyen, l'écrit est la matière première. Parfois, par manque de connaissances, nous ne pouvons pas donner du sens aux écrits de certains spécialistes, alors qu'ils nous parlent d'un sujet qui nous tient à Coeur. (El-Chahat, 2000: 31).

\subsubsection{Importance de la} compréhension écrite

D'après Sabri (2009: 50), la compréhension écrite n'est pas un acte gratuit. Elle est toujours orientée vers des objectifs précis:

- S'informer, collecter, classer, condenser, reformuler, l'information. La lecture, ce 
n'est pas découvrir quelque chose d'inconnu: c'est à partir de son expérience du monde et celle des autres cultures, faire des hypothèses sur ce qu'on lit.

- Les plaisirs de la lecture sont multiples. On lit pour savoir, pour comprendre, pour réfléchir. On lit aussi pour la beauté du langage, pour s'émouvoir et pour rêver.

- La lecture est une affaire publique: c'est un moyen de prise d'informations, donc une composante de l'acte social.

- La lecture est au centre du travail intellectuel, elle conditionne la réussite des études.

- Elle élargit et développe l'expérience de l'élève car elle offre des connaissances multiples dans tous les domaines.

- Elle ouvre les portes de la culture, de l'imagination, de la pensée critique, de la pensée créative et de la résolution des problèmes.

- Elle développe les différentes puissances et compétences.

- Elle entraîne les apprenants à consulter les dictionnaires, les références et à respecter les livres.

- Elle aide les apprenants à l'acquisition des valeurs, des critères et des attitudes morales, sociales et esthétiques.

- Elle développe les compétences de mémorisation et de compréhension.

- Elle s'intéresse au comment fonctionner et utiliser l'information dans la vie quotidienne.

\subsubsection{Types de la lecture}

Cuq et Gruca (2003: 163) exposent quatre types de la lecture:

- La lecture écrémage qui consiste à parcourir rapidement un texte et de manière non linéaire: ce survol du texte donne une idée globale de son contenu et c'est cette technique que nous pratiquons tous lorsque nous feuilletons un journal pour repérer quels sont les articles qui nous intéressent et que nous lirons par la suite.

- La lecture balayage qui permet de capter l'essentiel ou une information précise distribuée dans le texte par l'élimination rapide du reste, il s'agit d'une lecture sélective que l'on pratique au quotidien: parcourir un dépliant pour relever le lieu d'une activité, son horaire.

- La lecture critique qui demande une lecture intégrale d'un document et qui s'attache au détail et à la précision: elle peut entrainer le commentaire. 
- La lecture intensive ou studieuse qui vise à retenir le maximum d'informations et qui, par l'attention qu'elle réclame, peut se transformer en une quasi mémorisation du texte.

\subsection{5. Étapes de la lecture}

D'après Martin (1999: 34) il y a quatre étapes de la lecture

- L'avant lecture du texte: met en jeu les connaissances et les représentations initiales des apprenants.

- La lecture découverte ou balayage: est l'activité la plus évidente et qui correspond aux premières réactions du lecteur.

- La lecture sélective et organisée: est toujours une relecture soucieuse pour construire le sens.

- Les nouvelles perspectives de lecture du texte: d'autres textes permettent de sortir du carcan d'une démarche rigoureuse, de faire le point sur les acquis en les mettant au service de projets plus libres et plus personnels.

La lecture est le processus par laquelle on peut comprendre le texte écrite. Le processus de la lecture se divise en trois étapes:

\section{- Pré lecture}

C'est le moment de la mise en situation où l'étudiant prend connaissance de l'intention de lecture. Il est appelé à faire le point sur ses connaissances du sujet, de la structure ou du genre de texte écrite. De plus, l'étudiant lecteur commence à faire des prédictions, à émettre des hypothèses sur le texte écrite. C'est aussi à cette étape qu'il active ses connaissances antérieures et fait le lien avec son vécu et ses expériences personnelles.

\section{- Lecture}

C'est l'étape où l'étudiant lit le texte écrite et met en oeuvre les différentes stratégies qui lui permettront de gérer sa compréhension de son intention de lecture. Ainsi, il peut vérifier les hypothèses émises lors de l'étape de prélecture, organiser les informations présentées en les confrontant à ses connaissances antérieures et en venir à se poser de nouvelles questions qui ameneront d'autres hypothèses. L'étudiant lecteur fait continuellement un retour sur sa démarche de lecture afin de reconstruire le sens du texte lu.

\section{- Post lecture}

C'est l'étape où l'étudiant donne un objectif à sa démarche de compréhension. C'est aussi à cette étape qu'il réagit, analyse et évalue ses nouvelles connaissances pour se les approprier. Le processus de la lecture et de l'utiliser dans les 
autres situations d'apprentissage ElSafy (2005: 31-32).

\subsubsection{Notion de la compréhension écrite}

La compréhension est le but essentiel de la lecture, Elle permet à l'étudiant de relier une information à un modèle mental. Elle est un processus interactif qui met en relation un lecteur et un texte dans un contexte. Dans ce processus, le lecteur crée le sens en se servant à la fois du texte, de ses propres connaissances et de son intention de lecture. (Giasson, 2000: 5)

D'autre part, Giasson (2004: 22) voit que le lecteur construit le sens du texte à partir de l'information implicite et explicite $\mathrm{du}$ texte et de ses connaissances antérieures.

Quand à Turcotte (1992: 100), la compréhension écrite est une activité, cognitive et langagière, apprise, accomplie sous le contrôle d'un individu qui poursuit un but propre, elle est orientée vers la compréhension, c'est-à-dire la construction du sens du texte par le lecteur et elle est effectuée avec une aisance et une motivation variables qui en affectent le résultat. Les connaissances antérieures font partie du lecteur et influencent sa compréhension et le lecteur se caractérise par ses traits affectifs (son intérêt, son attitude, sa motivation au texte et son but de lecture) qui influencent sa compréhension.

Donc, on peut dire qu'on lit pour comprendre tout ce qui se passe autour de nous et la compréhension est le but final de la lecture. La compréhension lecturaleest la capacité à construire, à partir des données d'un texte et des connaissances antérieures, une représentation mentale cohérente de la situation évoquée par le texte. Le lecteur n'est pas toujours motivé face à la lecture parce qu'elle ne représente pas toujours un besoin: elle peut être imposée.

Giasson (2013: 227), indique que comprendre un texte, c'est s'en faire une représentation mentale cohérente en combinant les informations explicites, implicites qu'il contient à ses propres connaissances. Cette représentation est dynamique et cyclique. Elle se transforme et se complexifie au fur et à mesure de la lecture. Chaque fois que le lecteur rencontre un nouvel élément dans le texte, il doit décider de quelle façon l'intégrer à sa représentation du texte.

\subsubsection{Les variables de la compréhension écrite}

Giasson (2000), montre que la compréhension écrite est un processus interactif où 
interviennent trois variables: le lecteur, le texte et le contexte.

\section{- Le lecteur:}

Le lecteur constitue certainement la variable la plus complexe. Cette composante représente ce que le lecteur fait et ce qu'il sait. Le lecteur a des connaissances sur la langue et sur le monde. Ses connaissances représentent les structures cognitives qui influencent sa compréhension écrite. Ses connaissances, sur la langue, sont d'ordre phonologique (les phonèmes propres à sa langue), syntaxique (ordre des mots dans la phrase), sémantique (le sens des mots et les relations entre eux, son vocabulaire) et pragmatique (pratique). Les connaissances du lecteur sur le monde représentent "la théorie du monde" qu'il s'est construite et qui forme ses connaissances antérieures auxquelles il pourra rattacher les nouvelles informations fournies par le texte.

D'ailleurs, son attitude générale, ses goûts, ses besoins, la capacité de prendre des risques, le concept de soi en général comme lecteur et la peur de l'échec peuvent influencer la compréhension d'un texte.

\section{- Le texte:}

Les composantes du texte vont aussi jouer un rôle dans la compréhension écrite. On abordera le texte selon trois aspects principaux les intentions de

l'auteur, la forme du texte et le contenu du texte comme suit:

- Intention de l'auteur:

L'intention de l'auteur détermine en fait l'orientation des deux autres éléments, on reconnaît habituellement que l'auteur peut vouloir persuader, informer, distraire. C'est dans cette optique qu'on parlera de texte informatif, persuasif, incitatif. Plus l'auteur aura approfondi sa pensée et son argumentation, plus ses affirmations seront clairement formulées et précises, et par conséquent, la compréhension du texte s'en trouvera facilitée.

- La structure:

La structure fait référence à la façon dont les idées sont organisées dans un texte. Les structures peuvent aller des assertions simples aux structures les plus complexes à enchâssements multiples. Evidemment, les structures syntaxiques peuvent simplifier ou rendre difficile la compréhension du texte.

- Le contenu:

Le contenu est un facteur décisif de la compréhension. Il est à noter que la familiarité joue un rôle crucial dans la 
compréhension. Un lecteur ne comprendra pas le contenu spécialisé d'un sujet, même si le vocabulaire et la structure sont relativement faciles, si le contenu n'est pas familiarisé. Le contenu renvoie aux concepts, aux connaissances, au vocabulaire que l'auteur a décidé de transmettre.

\section{- Le contexte:}

Le contexte comprend toutes les conditions et les éléments qui ne font pas partie littéralement du texte et qui ne concernent pas directement les structures mais influent sur la compréhension du texte.

\subsubsection{Niveaux de la compréhension écrite}

La compréhension écrite résulte de la capacité de reconnaître et d'établir des liens entre le texte et ses connaissances et expériences antérieures. Cette capacité implique des processus cognitifs complexes.

La classification de Ministère de l'éducation de la Saskatchewan (2000)

Il existe quatre niveaux de compréhension écrite: la compréhension littérale, la compréhension inférentielle ou interprétative, la compréhension critique ainsi que la compréhension créative.

Les habiletés de ces quatre niveaux de compréhension sont organisées selon la hiérarchie de la taxonomie de Bloom comme suit:

\section{- La compréhension littérale:}

C'est comprendre les informations ou les idées données de façon explicite par l'auteur ou par l'auteure dans un texte. L'élève repère des informations, des idées ou des situations apparaissant clairement dans le texte.

\section{- La compréhension inférentielle ou interprétative:}

C'est comprendre des informations implicites supplémentaires. Le lecteur ou la lectrice les découvre par déduction à partir de ses structures cognitives. L'élève trouve des informations qui ne sont pas clairement exprimées dans le texte.

\section{- La compréhension critique:}

Le lecteur ou la lectrice évalue l'exactitude du texte lu à la lumière de ses connaissances. Il va porter un jugement sur le texte.

\section{- La compréhension créative:}

C'est le niveau de compréhension qui permet au lecteur ou à la lectrice d'appliquer les différentes significations trouvées dans une lecture, à sa vie personnelle. (cité par Fouad 2014: 49).

\subsubsection{Difficultés de la compréhension}


Barret (2006: 40), détermine les difficultés de la compréhension comme suit:

\section{- Difficultés linguistiques :}

- Vocabulaire dense et complexe

\section{- Difficultés textuelles :}

- Mémoriser la chaîne énumérative des personnages, des actions

- Extraire le thème du texte (repérer l'élément déclencheur, la quête du personnage principal)

- Localiser un indice important

- Verbaliser l'implicite: repérer les informations sûres et directes, celles obtenues par déduction, les informations manquantes, faire des liens de causalité...

- Repérer la complémentarité texte/image, les décalages texte/image

- Repérer comment la typographie peut matérialiser un point de vue

\section{- Difficultés culturelles:}

- Etablir des liens entre les connaissances antérieures et les informations du texte

- Accéder à l'intertextualité

- Proposer une interprétation argumentée

\section{- Difficultés métacognitives:}

- Vérifier, réfléchir sur ce qui a été compris, ce qui a permis de comprendre

- Déterminer le moment où on perd la compréhension

- Dépasser le stade des remarques ponctuelles et parcellaires

\section{Deuxième axe: la stratégie} d'auto-questionnement

\subsection{Notion de la stratégie} d'auto-questionnement

Selon Théorêt et al. (2004), cité par Viola (2000: 2), enseigner l'auto-questionnement permet aux élèves de se rendre compte des stratégies métacognitives qu'ils doivent mettre en place pour autogérer leur compréhension en lecture.

Selon Théorêt et al. (2004), cité par Viola (2000: 2), l'autoquestionnement est une des stratégies métacognitives qui aide les étudiants à s'autoquestionner avant, pendant et après la lecture d'un texte. Ces questions peuvent être catégorisé d'ordre cognitifs, affectifs ou métacognitifs.

\subsection{Types de questions}

Viola (2000: 2-3), précise que les questions peuvent être catégorisées d'ordre cognitifs, affectifs ou métacognitifs. Les prochains tableaux donne des 


\begin{tabular}{l}
\hline \hline $\begin{array}{l}\text { exemples de dype d'autoquestionnement: } \\
\text { Avant la lecture }\end{array}$ \\
\begin{tabular}{|l|l|}
\hline \multicolumn{1}{|c|}{ Types de questions } & \multicolumn{1}{c|}{ Exemples } \\
\hline $\begin{array}{l}\text { Les questions d'ordre } \\
\text { métacognitifs }\end{array}$ & $\begin{array}{l}\text { Ex: Pour faciliter la compréhension de } \\
\text { ce texte, qu'est-ce que je dois faire } \\
\text { avant de commencer à lire? }\end{array}$ \\
\hline Les questions d'ordre affectifs & $\begin{array}{l}\text { Ex: Est-ce que je veux m'engager dans } \\
\text { la lecture de ce texte }\end{array}$ \\
\hline Les questions d'ordre cognitifs & $\begin{array}{l}\text { Ex: Qu'est-ce que je sais déjà au sujet } \\
\text { de cette lecture? }\end{array}$ \\
\hline
\end{tabular}
\end{tabular}

\section{Pendant la lecture}

\begin{tabular}{|l|l|}
\hline \multicolumn{1}{|c|}{ Types de questions } & \multicolumn{1}{c|}{ Exemples } \\
\hline $\begin{array}{l}\text { Les questions d'ordre } \\
\text { métacognitifs }\end{array}$ & $\begin{array}{l}\text { Ex: est-ce que je comprends? } \\
\text { Qu'est-ce que je ne comprends pas ? }\end{array}$ \\
\hline Les questions d'ordre affectifs & Ex: Est-ce que j'aime ce que je lis? \\
\hline Les questions d'ordre cognitifs & $\begin{array}{l}\text { Ex: Je dois me poser des questions } \\
\text { sur les détails du texte }\end{array}$ \\
\hline
\end{tabular}

\section{Après la lecture}

\begin{tabular}{|l|l|}
\hline \multicolumn{1}{|c|}{ Types de questions } & \multicolumn{1}{c|}{ Exemples } \\
\hline Les questions d'ordre métacognitifs & Ex: Qu'est-ce que j'ai compris \\
\hline Les questions d'ordre affectifs & $\begin{array}{l}\text { Ex: Est-ce que je suis satisfaite des } \\
\text { apprentissages que j'ai effectués }\end{array}$ \\
\hline Les questions d'ordre cognitifs & $\begin{array}{l}\text { Ex: Je dois me poser des questions } \\
\text { sur le texte dans son ensemble }\end{array}$ \\
\hline
\end{tabular}

Les questions jouent un rôle important dans le travail sur la compréhension mais très souvent l'enseignant cherche à savoir si l'élève a compris plutôt que de l'aider par le biais des questions à mieux comprendre le texte. Barret (2006: 13) distingue deux types de questions :
- Questions d'évaluation dont la réponse se trouve explicitement dans le texte, il s'agit souvent de questions fermées : quel est le nom des personnages? où et quand se passe l'histoire ?...

Questions d'enseignement : elles permettent à l'élève d'évoluer dans sa compréhension car elles l'amènent à réfléchir sur la réponse. 
Là, les questions sont ouvertes et elles permettent de saisir l'articulation du texte, de travailler l'implicite et d'apporter un jugement critique.

\section{3. Étapes de la stratégie d'auto-questionnement}

Viola (2000: 3), propose la démarche en trois temps pour faciliter la compréhension de textes et aider les lecteurs à intéragir avec les textes. C'est la démarche d'avant, pendant et après lecture.

\section{Avant la lecture:}

Permet d'activer les connaissances des étudiants, de faire des prédictions sur le texte et de préciser l'intention de lecture.

\section{Pendant la lecture:}

Permet de vérifier les prédictions, d'en établir des nouvelles, de comprendre d'interpréter. ... etc.

\section{Après la lecture:}

Permet de vérifier les prédictions, de revenir sur l'intention de lecture, de réagir, de prolonger l'expérience de lecture. ...etc.

\section{Avantages de la stratégie d' autoquestionnement}

$\mathrm{La}$

d'autoquestionnement

l'apprentissage

stratégie

encourage autonome, apprendre a poser de questions et mettre l'accent sur le principe d'auto apprentissage. Viola (2000: 4), voit que/qu':
- La

stratégie

d'autoquestionnement permet le développement cognitif et métacogntif de l'élève.

- Elle permet la construction de sa propre représentation du texte.

- Cette stratégie d'enseignement permet aux étudiants de mieux réviser des textes que ceux qui ne l'utilisent pas. De plus, les stratégies cognitives d'autoquestionnement sont utiles chez les étudiants qui ont un faible rendement scolaire.

- Elle permet aux étudiants d'effectuer de meilleurs apprentissages.

14. Rôle de l'étudiant dans la stratégie d' autoquestionnement

La stratégie d'autoquestionnement est une stratégie d'enseignement qui permet l'apprentissage dans une perspective cognitive. Dans cette stratégie, c'est l' étudiant qui exerce son apprentissage puisque c'est lui qui s'autoquestionne et cherche des réponses pour satisfaire son besoin d'apprentissage.

L'enseignant ne peut pas intervenir dans le cerveau de ses élèves pour y insérer de nouveaux éléments de savoir.

Les connaissances que chacun possède ne sont accessibles 
qu'à lui seul. C'est donc l'étudiant qui doit créer lui-même de nouvelles structures de connaissance. Donc l'autoquestionnement permet à l' étudiant devient au coeur de son apprentissage. (Viola, 2000: 4)

Donc l'étudiant joue le rôle essentiel dans la stratégie d' autoquestionnement où il organise son apprentissage

\section{Résultats de la recherche}

Cette recherche vise à vérifier l'efficacité de la stratégie d'autoquestionnement pour le développement les compétences de la compréhension des écrits chez les étudiants des écoles officielles françaises des langues. Pour cela cette recherche comprend cette hypothèse «Il y a une différence significative statistiquement entre les moyennes des étudiants de l'échantillon au pré-test et au post test en faveur du post-test» pour s'assurer l'efficacité de cette stratégie la chercheuse a appliqué la recherche le pré/post-test sur un échantillon des étudiants de la première année secondaire car les membres de l'échantillon sont limite (9 étudiants) seulement.

Le pré-test assure que les étudiants ont une faiblesse à comprendre un texte. Après l'enseignement de l'unité reformulée selon la stratégie d'auto-questionnement les résultats ont conduit à la vérification de l'hypothèse de la recherche.

En effet, les résultats de la recherche ont mis l'accent sur l'efficacité de la stratégie d'autoquestionnement sur le développement de certaines compétences de la compréhension des écrits.

Enfin, la chercheuse propose un certain nombre de suggestions et de recommandations qui peuvent conduire au développement des compétences de la compréhension des textes en français chez les étudiants du cycle secondaire. 
Tableau $\mathbf{N}^{\mathbf{0}}$ (1) Valeur $\mathrm{Z}$ et sa signification statistique des différences entre les moyens des rangs de notes de l'échantillon aux pré/post-tests des compétences de la compréhension écrite

\begin{tabular}{|c|c|c|c|c|c|c|c|}
\hline \multirow{3}{*}{$\begin{array}{c}\text { Comp. } \\
\text { A1 } \\
\end{array}$} & \multicolumn{2}{|c|}{ Mean rank } & \multicolumn{2}{|c|}{ Sum of ranks } & \multirow{2}{*}{$\mathbf{Z}$} & \multirow{2}{*}{\multicolumn{2}{|c|}{ Sign. }} \\
\hline & W (-) & $\mathrm{W}(+)$ & $\mathrm{W}(-)$ & $\mathrm{W}(+)$ & & & \\
\hline & 0 & 4 & 0 & 28 & 2.46 & 0.014 & Sign. \\
\hline $\mathrm{A} 2$ & 0 & 3.5 & 0 & 21 & 2.45 & 0.014 & Sign. \\
\hline $\mathbf{A}$ & $\mathbf{0}$ & 5 & 0 & 45 & 2.72 & 0.007 & Sign. \\
\hline $\mathrm{B} 1$ & 0 & 3 & 0 & 15 & 2.24 & 0.025 & Sign. \\
\hline $\mathrm{B} 2$ & 0 & 2.5 & 0 & 10 & 2.00 & 0.046 & Sign. \\
\hline B3 & 0 & 3 & 0 & 15 & 2.24 & 0.025 & Sign. \\
\hline B4 & 0 & 3.5 & 0 & 21 & 2.45 & 0.014 & Sign. \\
\hline B5 & 0 & 4.5 & 0 & 36 & 2.83 & 0.005 & Sign. \\
\hline B6 & 0 & 3.5 & 0 & 21 & 2.45 & 0.014 & Sign. \\
\hline B7 & 0 & 2.5 & 0 & 10 & 2.00 & 0.046 & Sign. \\
\hline B8 & 0 & 3.5 & 0 & 21 & 2.45 & 0.014 & Sign. \\
\hline B & 0 & 5 & 0 & 45 & 2.68 & 0.007 & Sign. \\
\hline $\mathrm{C} 1$ & 0 & 2.5 & 0 & 10 & 2.00 & 0.046 & Sign. \\
\hline $\mathrm{C} 2$ & 0 & 2.5 & 0 & 10 & 2.00 & 0.046 & Sign. \\
\hline $\mathrm{C} 3$ & 0 & 3 & 0 & 15 & 2.24 & 0.026 & Sign. \\
\hline $\mathrm{C} 4$ & 0 & 2.5 & 0 & 10 & 2.00 & 0.046 & Sign. \\
\hline $\mathrm{C} 5$ & 0 & 3 & 0 & 15 & 2.24 & 0.025 & Sign. \\
\hline $\mathrm{C} 6$ & 0 & 3 & 0 & 15 & 3.00 & 0.003 & Sign. \\
\hline $\mathrm{C} 7$ & 0 & 5 & 0 & 45 & 2.45 & 0.014 & Sign. \\
\hline $\mathrm{C} 8$ & 0 & 3.5 & 0 & 21 & 2.45 & 0.014 & Sign. \\
\hline $\mathrm{C}$ & 0 & 5 & 0 & 45 & 2.68 & 0.007 & Sign. \\
\hline D1 & 0 & 3.5 & 0 & 21 & 2.45 & 0.014 & Sign. \\
\hline $\mathrm{D} 2$ & 0 & 3.5 & 0 & 21 & 2.45 & 0.014 & Sign. \\
\hline D & 0 & 3.5 & 0 & 21 & 2.45 & 0.014 & Sign. \\
\hline Total & 0 & 5 & 0 & 45 & 2.67 & 0.008 & Sign. \\
\hline
\end{tabular}

Les chiffres du tableau précédent indiquent que/qu':

- Il existe des différences statistiques significatives entre les moyennes de notes des étudiants de l'échantillon au post-test par rapport aux compétences de la compréhension des écrits, cela prouve que le niveau des 
étudiants est plus élevé que celui du pré-test.

post-test en ce qui concerne les

La dispersion de notes des compétences de la étudiants de l'échantillon au

compréhension écrite indique que leur niveau est plus élevé.

Tableau $\mathbf{N}^{\mathbf{0}}$ (2) Pourcentage des notes des étudiants par rapport aux compétences principales et la note totale au pré-test

\begin{tabular}{|c|c|c|c|c|c|}
\hline Etudiant & $\begin{array}{c}\mathbf{1}^{\text {ère }} \\
\text { compétence }\end{array}$ & $\begin{array}{c}\mathbf{2}^{\text {ième }} \\
\text { compétence }\end{array}$ & $\begin{array}{c}\mathbf{3}^{\text {ième }} \\
\text { competence }^{\text {compere }}\end{array}$ & $\begin{array}{c}\mathbf{4}^{\text {ième }} \\
\text { compétence }\end{array}$ & $\begin{array}{c}\text { Note } \\
\text { totale }\end{array}$ \\
\hline 1 & 50 & 75 & 62.5 & 0 & 60 \\
\hline 2 & 0 & 62.5 & 37.5 & 0 & 40 \\
\hline 3 & 25 & 12.5 & 62.5 & 0 & 32.5 \\
\hline 4 & 0 & 12.5 & 50 & 0 & 25 \\
\hline 5 & 50 & 0 & 37.5 & 0 & 15 \\
\hline 6 & 25 & 12.5 & 37.5 & 0 & 22.5 \\
\hline 7 & 0 & 50 & 50 & 0 & 40 \\
\hline 8 & 100 & 87.5 & 62.5 & 0 & 70 \\
\hline 9 & 100 & 12.5 & 50 & 0 & 35 \\
\hline
\end{tabular}

Le tableau précédent

écrite au pré-test. Les notes des indique que les étudiants de l'échantillon ont une faiblesse en ce qui concerne les compétences principales de la compréhension étudiants montrent que la quatrième compétence est la plus faible entre les compétences où ils ont obtenu $0 \%$.

Tableau $\mathbf{N}^{\mathbf{0}}$ (3) Pourcentage des notes des étudiants par rapport aux compétences principales et la note totale au post-test

\begin{tabular}{|c|c|c|c|c|c|}
\hline Etudiant & $\begin{array}{c}\mathbf{1}^{\text {ère }} \\
\text { compétence }\end{array}$ & $\begin{array}{c}\mathbf{2}^{\text {ième }} \\
\text { compétence }\end{array}$ & $\begin{array}{c}\mathbf{3}^{\text {ième }} \\
\text { compétence }\end{array}$ & $\begin{array}{c}\mathbf{4}^{\text {ième }} \\
\text { compétence }\end{array}$ & $\begin{array}{c}\text { Note } \\
\text { totale }\end{array}$ \\
\hline 1 & 100 & 100 & 100 & 100 & 100 \\
\hline 2 & 100 & 100 & 100 & 100 & 100 \\
\hline 3 & 100 & 100 & 100 & 100 & 100 \\
\hline 4 & 100 & 100 & 100 & 100 & 100 \\
\hline 5 & 100 & 100 & 100 & 100 & 100 \\
\hline 6 & 100 & 100 & 100 & 100 & 100 \\
\hline 7 & 50 & 100 & 75 & 0 & 75 \\
\hline 8 & 100 & 100 & 100 & 0 & 90 \\
\hline 9 & 100 & 75 & 87.5 & 0 & 75 \\
\hline
\end{tabular}


Le tableau précèdent montre le pourcentage des notes des étudiants par rapport aux compétences principales et la note totale au post-test de la compréhension écrite, dont on voit qu'une grande amélioration entre les notes des étudiants. On remarque aussi une amélioration aux compétences de la compréhension écrite particulièrement la quatrième compétence sauf le septième, le huitième et le neuvième étudiant ont une faiblesse à cette compétence où ils ont obtenu $0 \%$.

\section{Interprétation des résultats de la recherche}

Selon les résultats de la correction du post-test après l'enseignement de l'unité reformulée par la stratégie d'autoquestionnement à l'échantillon de la recherche, la chercheuse a remarqué un progrès au niveau des étudiants de l'échantillon en ce qui concerne les compétences de la compréhension écrite.

Ce résultat peut nous amener à constater que la stratégie d'autoquestionnement est efficace pour développer les compétences de la compréhension écrite chez les étudiants de la première année secondaire aux écoles officielles françaises des langues.

\section{Recommandations de la recherche}

Après avoir présenté les résultats et l'interprétation de la recherche, il est convenable de proposer quelques recommandations en vue d'être prochainement prises en compte:

- Encourager les étudiants à lire les différents textes dans les différents cycles de l'enseignement afin de développer leurs compétences en compréhension.

- Favoriser le climat adéquat qui réalise l'apprentissage stratégique flexible et créatif.

- Utiliser la stratégie d'autoquestionnement dans le développement des compétences de lecture aux domaines différents tels que: les sciences, l'histoire, les langues...etc.

- Organiser des conférences et des cours aux enseignants afin de les entrainer à utiliser les stratégies de métacognition, notamment la stratégie d'autoquestionnement au domaine d'enseignement/apprentissage de lecture.

- Attirer l'attention des enseignants et des apprenants sur la compréhension en lecture comme une base essentielle de toute lecture.

- Insister sur les activités d'apprentissage qui favorisent 
l'autonomie de l'apprenant dans l'acte pédagogique et sur les activités qui préconisent sa participation positive avec ses camarades en classe.

- Motiver les apprenants d'être au centre du processus de l'apprentissage et non seulement un transmetteur d'information et un récepteur.

\section{Suggestion de la recherche}

Selon les résultats de cette étude, on peut présenter les suggestions suivantes:

- Réappliquer la stratégie d'autoquestionnement sur d'autres cycles.

- Réappliquer la stratégie d'autoquestionnement afin de développer d'autres sortes de lecture comme la lecture silencieuse, rapide, critique. ...etc.

- Vérifier le rapport entre la stratégie d'auto-questionnement et les autres compétences langagières.

- Vérifier l'effet de quelques stratégies de métacognition sur la réflexion créative et sur les attitudes des apprenants envers la langue française comme une langue étrangère.

\section{Conclusion}

Les résultats de la recherche affirment que la participation des étudiants à travers la stratégie d'auto-questionnement a une grande influence sur l'accroissement de leur compétence lecturale, ainsi que sur l'interaction en classe, entre eux ou avec l'enseignant.

\section{Bibliographie}

\section{Références en francais:}

Abdel- Ghany, S-A. (2009). La lecture entre le décodage et la compréhension. Cahier du CRISCO, 28.

Abdel-Aal, M. (2016). Efficacité de la stratégie de l'enseignement réciproque pour le développement des compétences de la compréhension des textes lus et l'attitude envers la langue française chez les étudiants $\mathrm{du}$ cycle secondaire d'Al Azhar, thèse de magistère, Faculté de pédagogie Université de Mansourah.

Afifi, A. (2018). Efficacité d'un programme basé sur l'enseignement différencié sur le développement des compétences de la compréhension en lecture chez les étudiants de la section de français. Thèse de Magistère, Faculté de Pédagogie - Université d'Al Azhar.

Alsafy, C. (2010). Efficacité d'un programme proposé basé sur l'approche du processus de l'écriture pour le 
développement de quelques compétences de la production écrite et les attitudes des élèves du cycle secondaire. Thèse de Doctorat, Faculté de jeunes filles - Université d'Ain Chams.

Barret, E. et al. (2006). Enseigner la comprehension de textes littéraires. Tiré de http://paf.acdijon.fr/doc_stagi aires/goigoux\%2520thomazet lecture\% $\% 2520$ comprehension. pdf.

Cuq, J.-P. (2003). Dictionnaire de didactique du français langue étrangère et seconde. Paris: CLE International.

Cuq, J. et Gruca, I. (2003). Cours de didactique du français langue étrangère et seconde. France: Presses Universitaires de Grenoble.

El-Chahat, G. (2000). Efficacité du programme proposé pour le développement des compétences de lecture d'étude chez les étudiants des départements de français des facultés de pédagogie. Thèse de Doctorat, Faculté de pédagogie - Université de Mansourah.

Fouad, R. (2014). Efficacité de l'emploi de la stratégie des cartes sémantiques sur le développement des compétences de la compréhension lecturale chez les étudiants de la faculté de pédagogie-section de français. Thèse de Magistère. Faculté de pédagogie - Université de Mansourah.

Giasson, J. (2000). La compréhension en lecture, Québec: Gaëtan Morin.

Giasson, J. (2004). La lecture de la théorie à la pratique, Paris: De Boeck.

Giasson, J. (2013). La lecture: apprentissage et difficulté, $1^{\text {ère }}$ édition., 3e tirage, Bruxelles: De Boeck.

Martin, M. (1999). Jeux pour lire: apprendre à lire-écrire avec des albums. Paris: Hachette.

Pierre, R. (2003). L'enseignement de la lecture au Québec de 1980 à 2000: fondements historiques, épistémologiques et scientifiques. Revue des sciences de l'éducation, $\mathrm{XXI} / 1$.

Sabri, N. (2009). Efficacité d'un programme propose à la lueur de l'Approche des histoires sur le développement de quelques compétences de la compréhensions lecturale en langue française et de valeurs morales chez les élèves du cycle préparatoire, Thèse de doctorat, Faculté de Jeunes Filles - Université d'Ain Chams. 
Soleiman, R. (1996). Efficacité du programme proposé pour le développement de certaines compétences auditives chez les étudiants des facultés de pédagogies section de français. Thèse de Magistère, Faculté de pédagogie Université de Mansourah.

Turcotte, C. (1992). Un cadre théorique sur la lecture et un modèle du lecteur (conçus en vue de l'évaluation diagnostique du lecteur cégépien). Mémoire de maîtrise - Université de Sherbrooke.

Viola, S. (2000). L'autoquestionnement en lecture. Québec français, 118, 63-66.

\section{Références en arabe}

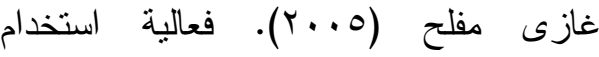

$$
\begin{aligned}
& \text { استر اتيجية التعلم التعاونى فى تتمية بعض }
\end{aligned}
$$

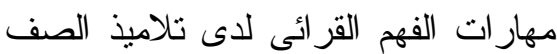

$$
\begin{aligned}
& \text { الأول الثانوى. مجلة جامعة دمشق. كلية } \\
& \text { التربية. العدد الثانى. المجلد الب. }
\end{aligned}
$$

